\title{
An acoustic trade-off chart for the design of multilayer acoustic packages
}

\author{
Hugues Pichon ${ }^{1}$, Elsa Piollet ${ }^{1}$, Annie Ross ${ }^{1}$
}

\section{Abstract}

This paper presents a novel trade-off chart to support the design of multilayer acoustic packages. In this multi-objective problem, a designer has to specify a combination of layers from a set of available acoustic materials and thicknesses. Material types may include porous, mass-weighted, facing, among others. The combination must meet requirements in terms of sound absorption, sound transmission loss, cluttering, mass, etc. While predictions and analyses can be made on predetermined multilayer acoustic packages using the transfer matrix method, statistical energy analysis, finite elements methods or modal analysis, comparing a large number of possible combinations is cumbersome. On the other hand, optimization methods can be used to identify optimal thicknesses or material properties for a given layer combination, but the obtained solution may not be industrially relevant since, in general, only a limited set of acoustic materials and layer thicknesses exist commercially. In this paper, a new design methodology is proposed, which takes into account only the feasible combinations and provides guidelines for compromises between different performance parameters. The three-step methodology is demonstrated through a case study inspired by the automotive industry. First, relevant categories of layer configurations are defined, and following these patterns, all possible combinations of materials from a given inventory are calculated and stored in a database. Then, for selected performance parameters, the Pareto set of "better combinations" is identified. Finally, the "better solutions" are displayed on a trade-off chart through utility functions that allow weighing the different performance parameters. The tool developed for doing so is applied to the case study, and two example situations are presented. For each situation, the trade-off chart provides several suitable solutions, which are discussed. The use of this new tool effectively induces gains of time at the early stage of design, when it is most crucial.

\section{Keywords}

design; multilayer; acoustic package; Pareto set; multi-objective

1 - Laboratory for Acoustics and Vibration Analysis (LAVA), Center for Applied Research on Polymers and Composites (CREPEC), Department of Mechanical Engineering, École Polytechnique de Montréal, CP 6079, Station Centre-ville, Montréal; QC; H3C 3A7, Canada 


\section{Une carte de compromis acoustique pour la conception de solutions acoustiques multicouches}

Hugues Pichon ${ }^{1}$, Elsa Piollet ${ }^{1}$, Annie Ross ${ }^{1}$

\section{Résumé}

Ce papier présente une nouvelle carte de compromis pour aider à la conception de solutions acoustiques multicouches. Dans ce problème multi-objectif, un concepteur doit spécifier une combinaison de couches à partir d'un jeu de matériaux et d'épaisseurs disponibles. Les types de matériaux peuvent inclure des matériaux poreux ou lestés, ou des couches de protections, par exemple. La combinaison doit répondre à des exigences d'absorption acoustique, de perte par transmission, d'encombrement, de masse, etc. Bien que des prédictions et des analyses puissent être réalisées pour une solution acoustique multicouche donnée en utilisant la méthode des matrices de transfert, l'analyse statistique énergétique, la méthode des éléments finis ou l'analyse modale, comparer un grand nombre de combinaisons possibles est complexe et coûteux. D'autre part, les méthodes d'optimisation peuvent être utilisées pour identifier les épaisseurs et les propriétés matériau optimales pour une combinaison de couches données, mais la solution obtenue peut ne pas être pertinente d'un point de vue industriel puisque, de manière générale, les matériaux acoustiques et les épaisseurs disponibles commercialement sont limités. Dans ce papier, une nouvelle méthodologie de conception est proposée, qui prend en compte uniquement les combinaisons réalisables et qui fournit des lignes directrices pour des compromis entre différents critères de performance. La méthodologie, composée de trois étapes, est présentée à travers un cas d'application inspiré par l'industrie automobile. Dans un premier temps, des catégories de configurations multicouches pertinentes sont définies, et toutes les combinaisons possibles respectant ces configurations avec un inventaire de matériaux donné sont calculées et stockées dans une base de données. Puis, pour des critères de performance choisis, le front de Pareto des «meilleures combinaisons » est identifié. Finalement, les « meilleures solutions » sont représentées sur une carte de compromis à l'aide de fonctions d'utilité qui permettent de pondérer différents critères de performance. L'outil développé est appliqué au cas d'application, et deux situations types sont présentées. Pour chaque situation, la carte de compromis fournit plusieurs solutions pertinentes, qui sont discutées. L'utilisation de ce nouvel outil permet un gain de temps aux étapes initiales de la conception, là où cela est le plus crucial.

\section{Mots-clés}

conception; multicouche; solution acoustique; front de Pareto; multi-objectif

1 - Laboratoire d'Analyse Vibratoire et Acoustique (LAVA), Centre de recherche sur les systèmes polymères et composites à haute performance (CREPEC), Département de génie mécanique, École Polytechnique de Montréal, CP 6079, Station Centreville, Montréal; QC; H3C 3A7, Canada 


\section{Introduction}

Noise is considered a public health problem [1], and efforts are made to find innovative solutions to reduce noise levels. Multilayer acoustic packages are a usual solution to noise control problems in many fields of application such as the automotive industry [2] and the aerospace industry [3], as they can have a "superior sound suppression capability" [4]. Multilayer materials can achieve properties unmatched by monolithic materials: the vast number of possible material and layer combinations allows for a good adaptability to various requirements. However, the choice of a combination fitted to a given acoustic problem is not obvious. One typical objective is to maximize package sound transmission loss, i.e. its capacity to block sound. Another important objective is to maximize package absorption, i.e. its capacity to absorb sound without reflection. Transmission loss and absorption are often conflicting, since a panel with high transmission loss generally tends to reflect sound. At the design stage, the designer is often faced with a lack of guidelines to balance objectives.

Several methods exist to predict the performance of multilayer acoustic packages. The transfer matrix method (TMM) [5] was developed to predict both absorption and transmission loss of multilayer acoustic panels. Since it is easily translated into a code and automatable, the method is used to investigate new acoustic materials and their acoustic performance in a wide range of studies and applications ([6], [7] for instance). Statistical energy analysis [8] can be used to predict acoustic performance by an energy approach. Modal analysis [9] is used to predict transmission loss for finite vibrating structures. Finite element methods are used in many studies [10-12] as a prediction tool for acoustic materials, especially when a complex geometry prevents the use of the TMM. All these methods allow for calculating the acoustic performance of given multilayer packages, but are insufficient for choosing the "best" solution to a specific noise problem. Combined with these prediction tools, designers need specific tools to compare, analyze and make compromises between all the possible solutions to a given problem.

Various methods have been proposed to design acoustic packages. Better performance can be identified by directly comparing predicted performance of possible solutions [13], [14]. Optimization methods such as topology optimization [15], genetic algorithms [16] or bi-objective Pareto approach [17] were also used to find the best thicknesses or material properties for each layer in the early design process.

In the industrial reality, material layers (especially their microstructures and thicknesses) are not custommade, and thus designers only work with a limited set of layers at their disposal to build a multilayer package. From this set of layers, many combinations can be assembled. Thus, a practical tool must take into account many configurations and industrial constraints, which is not possible with existing methods. Hence, there is a need for new design tools adapted to multilayer acoustic packages. The present paper aims to propose a tool and methodology to guide the early stages of design, involving multiple objectives and trade-off between performance goals. This tool is a material selection method based on Sirisalee et al. [18], adapted to the design of multilayer acoustic packages and meeting industrial constraints.

First, usual methods for choosing multilayer acoustic packages are outlined, and other design methods in various fields are considered. Then, a case study is defined with the intent of reflecting industrial constraints. Next, a new tool for assessing performance of multilayer acoustic packages is introduced. Finally, this new tool is applied to the case study, leading to a general conclusion and discussion. 


\section{Usual selection process for multilayer acoustic packages}

\subsection{Direct comparison between predicted properties}

A simple way to choose an acoustic package is to directly compare predicted properties. Absorption coefficient or transmission loss are usually plotted against the frequency, for different configurations of materials. This allows direct visual comparison between configurations ([6], [10] or [11] for instance).

Direct comparison of predicted properties can help choosing between different solutions, including different layer configurations. However, they are restricted to specific cases where a small number of material combinations is studied. Therefore, this method is not suited to industrial constraints where all combinations from a set of available layers have to be investigated. A visual comparison of many solutions in the same plot would be impossible. This method is also not adapted to trade-off issues, which require a designer to navigate between many possible solutions.

\subsection{Optimization algorithms for acoustic materials}

Optimization algorithms can be used to reach performance goals in a more systematic manner than through direct comparison. This section presents some algorithms used specifically in acoustic design. Tanneau et al. [16] used a genetic algorithm to minimize the difference between the transmission loss spectrum of a package and an objective spectrum, while taking into account the weight of the package. Cameron et al. [19] used a two-step optimization procedure on a sandwich structure. Both acoustic properties and structural properties were considered. First the topology was optimized using a bidirectional algorithm, then the acoustic and structural properties were optimized by varying the materials and their thicknesses. Inoue et al. [20] used a preference-set design method to obtain different design solutions adapted to multi-criteria constraints for multilayer acoustic packages. The method took into account 15 design variables including Biot's poroelastic parameters, and four performance parameters: absorption, transmission loss, mass and cost. However, the method applies only to one given multilayer combination, and the design variables used are intrinsic properties of materials and thicknesses of layers. Leite et al. [21] proposed a multi-objectives method using Pareto optimization, to design sandwich panels for both acoustic and mechanical performance. The method involves many material types for the core and skins, but is limited to only one configuration: the stacking order of layer types is fixed. Zhou et al. [17] used a bi-objective Pareto approach to optimize three different configurations of sandwich panels, focusing on transmission loss and surface density of the panels.

These studies reveal that optimization algorithms are powerful tools which are suited when a given configuration is defined. Therefore, they are efficient for case by case studies, but inappropriate for situations that involve a large number of configurations, and for fixed material inventory.

\subsection{Other methods in various fields}

In order to identify a method suitable for the problem at hand, material design methods employed in different fields can also be considered. Material property charts are a way to choose a material fitting specific requirements in mechanical design [22]. Usually, solutions are grouped into families with different property ranges. Property charts are a good way to visualize many solutions on a single chart and can be sufficient for meeting needs when only two properties are selection criteria. However, if there are more selection criteria, another way to make a trade-off between these performance goals is necessary. 
Moreover, for high numbers of solutions, a direct display into a scatter plot is not readable. Thus, a way to display only the more interesting solutions is needed.

Response surface methodology (RSM) was used in design optimization to improve performance of various systems. Avalle et al. [23] optimized the geometrical configuration of thin-walled tubes with RSM in the context of automotive safety. By varying geometrical parameters of tubes, the method successfully came up with a solution which was more performant in the event of an automotive crash. Like other optimization algorithms, RSM focuses on a single configuration and it provides only one optimized solution.

Sirisalee et al. [18] introduced a multi-criteria material selection method in engineering design, using a Pareto set. The method consists in sorting Pareto solutions with a utility function and then displaying the best solutions in a trade-off chart. The authors applied the method to two material selection cases: for a disk brake calliper, and for the casing of a mini-disk player. These cases focused on mechanical properties, thermal properties, mass, thickness and cost of materials.

Farag [24] introduced the weighted property method as a material selection tool. Each property (or performance parameter) is assigned a weight depending on its degree of importance. From these weights, a performance index can be calculated for each material. The material with the higher performance index is the chosen solution. Alternative solutions can be ranked and trade-off is made possible by varying property weights. This selection method is an interesting alternative to Sirisalee et al. [18], but does not display results on a visual chart.

In this paper, the method from Sirisalee et al. [18] is adapted to multilayer acoustic package selection in section 4 . This method was selected because it can be used with many configurations in accordance to the industrial reality: it provides multiple possible solutions in a visual chart, and the choice of a solution can be made by a trade-off between performance parameters.

\section{Case study}

A case study is presented here to illustrate a realistic design process. The acoustic package of interest is to be used in automotive applications, where it will be bonded to an existing metal panel in order to improve both transmission loss through that panel and absorption inside the cavity in which the package is placed. This cavity can be either the engine compartment or the passenger cabin. The goal is to choose the multilayer acoustic package with the best performance in terms of absorption and transmission, while keeping the surface density and the thickness of the package as low as possible. These objectives are conflicting: transmission loss is mainly associated with high surface density according to the "mass law" [25], and high absorption is mainly achieved by using thick absorptive layers. It is important to keep an overview of the possible solutions to a problem, so as to achieve a compromise between objectives. Hence, there is a need for guidelines rather than for a single optimal solution. This way, a designer has access to alternative solutions which could be more feasible from an industrial point of view.

In the present case, the multilayer package can be made of three types of layers:

- Porous layers, known for their absorption properties and widely used in acoustic design [26]. These layers are modelled using a poroelastic model based on the Biot theory [27] combined with a Johnson-Champoux-Allard model for the porous behaviour [5]. This model includes the deformation of porous layers, which is not negligible for structures involving barriers. Poroelastic 
materials are described with 8 parameters: Young's modulus $E$, Poisson's ratio $v$, density $\rho_{V}$, porosity $\phi$, resistivity $\sigma$, tortuosity $\alpha_{\infty}$ and characteristic lengths $\Lambda$ and $\Lambda^{\prime}$.

- Barriers: these layers are typically weighted layers, used for their high density. As described by the "mass law", such materials are effective for achieving high transmission loss [25]. Barriers are modelled as "impervious screens", as described by Allard [5], and are described with their surface density $\rho_{S}$.

- Facings: these layers are used on top of porous layers as a protection against the environment [28]. Facings are modelled as impervious screens, and are described with their surface density $\rho_{S}$.

From a modelling point of view, the incident acoustic field is assumed to be diffuse, and layers are assumed to have infinite in-plane dimensions. For purposes of simplicity and efficiency, the TMM is chosen to predict performance. However, absorption coefficient and transmission loss could also be predicted using SEA or FEM.

The following rules are defined to limit the scope of the case study, which is meant to be close to the reality of acoustic package design. A limited set of materials and layer thicknesses is available. Materials for each layer are presented in Table 1, along with their properties. Polyurethane and melamine foams are well-known acoustic materials which are widely used for their absorption properties [29, 30]. The facing is a polyester film, often known under the commercial name Mylar [31] and typically used as a facing over a layer of porous material $[30,32]$. The mass-loaded elastomer barrier is modelled with properties from the commercial general-purpose K-FONIK GK [33]. The materials chosen in this case study are widely used by original equipment manufacturers.

Table 1: Properties of layer materials

\begin{tabular}{|c|c|c|c|c|c|c|c|c|c|c|c|}
\hline Material & $\begin{array}{c}E \\
\left(\mathrm{~N} \cdot \mathrm{m}^{-2}\right)\end{array}$ & $v$ & $\begin{array}{c}\rho_{V} \\
\left(\mathrm{~kg} \cdot \mathrm{m}^{-3}\right)\end{array}$ & $\eta$ & $\phi$ & $\begin{array}{c}\sigma \\
\left(\text { N.s. } \mathrm{m}^{-4}\right)\end{array}$ & $\alpha_{\infty}$ & $\begin{array}{c}\Lambda \\
\left(10^{-6} \mathrm{~m}\right)\end{array}$ & $\begin{array}{c}\Lambda^{\prime} \\
\left(10^{-6} \mathrm{~m}\right)\end{array}$ & $\begin{array}{c}h \\
\left(10^{-3} \mathrm{~m}\right)\end{array}$ & $\begin{array}{c}\rho_{S} \\
\left(\mathrm{~kg} \cdot \mathrm{m}^{-2}\right)\end{array}$ \\
\hline \multicolumn{12}{|l|}{ Base plate } \\
\hline Aluminium & $7 \times 10^{10}$ & 0.33 & 2700 & 0.01 & - & - & - & - & - & 3 & - \\
\hline \multicolumn{12}{|l|}{ Porous layers } \\
\hline Melamine foam & $1.6 \times 10^{5}$ & 0.44 & 8.35 & 0.06 & 0.99 & 12600 & 1.0 & 78 & 192 & - & - \\
\hline $\begin{array}{l}\text { Polyurethane } \\
\text { foam }\end{array}$ & $8.45 \times 10^{5}$ & 0.3 & 31.16 & 0.1 & 0.96 & 32000 & 1.7 & 90 & 165 & - & - \\
\hline \multicolumn{12}{|l|}{ Facings } \\
\hline Polyester film & - & - & - & - & - & - & - & - & - & 0.127 & 0.1765 \\
\hline \multicolumn{12}{|l|}{ Barriers } \\
\hline $\begin{array}{l}\text { Mass-loaded } \\
\text { elastomer }\end{array}$ & - & - & - & - & - & - & - & - & - & 2.54 & 4.882 \\
\hline
\end{tabular}


In the real industrial case, layer thicknesses are not custom-made. Therefore, layer thicknesses are limited to a set of available values. For porous layers, thickness is: 2, 5, 10, 15, 20, 25, 30, 40 or $50 \mathrm{~mm}$. Barriers and facings have a single thickness $h$, given in Table 1, though up to three barriers can be stacked sequentially to add weight.

The multilayer acoustic package is applied to a $3 \mathrm{~mm}$ aluminium base plate, modelled as a Kirchhoff plate. The base plate is the initial system before applying the solution, and serves as a reference. From industrial experience, some multilayer topologies are particularly suitable for acoustic packages. Table 2 and Figure 1 present the seven topology categories identified for this study. Examples of possible acoustic packages for the case study are illustrated in Figure 2.

Table 2: Categories and quantity of multilayer structures used in the case study

\begin{tabular}{llc}
\hline Category & \multicolumn{1}{c}{ composition } & number of structures \\
\hline base layer & base layer & 1 \\
category A & base layer + porous layer & 18 \\
category B & base layer + porous layer + facing & 18 \\
category C & base layer + porous layer + barrier & 54 \\
category D & base layer + porous layer + barrier + porous layer & 972 \\
category E & base layer + porous layer + barrier + porous layer + facing & 972 \\
category F & base layer + porous layer + barrier + porous layer + barrier & 2916 \\
\hline
\end{tabular}

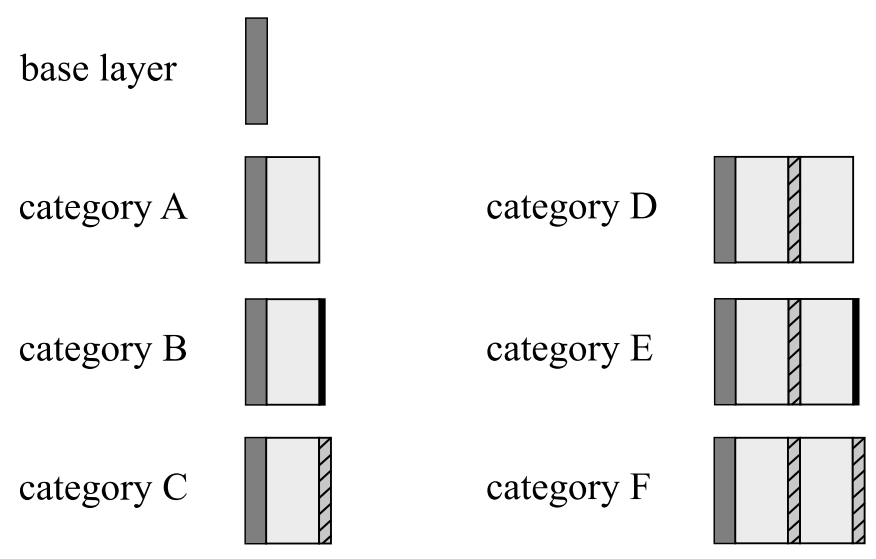

Figure 1: Categories of multilayer structures considered in the present work : light grey layers are porous layers, grey hatched layers are barriers, thin black layers are facings. 

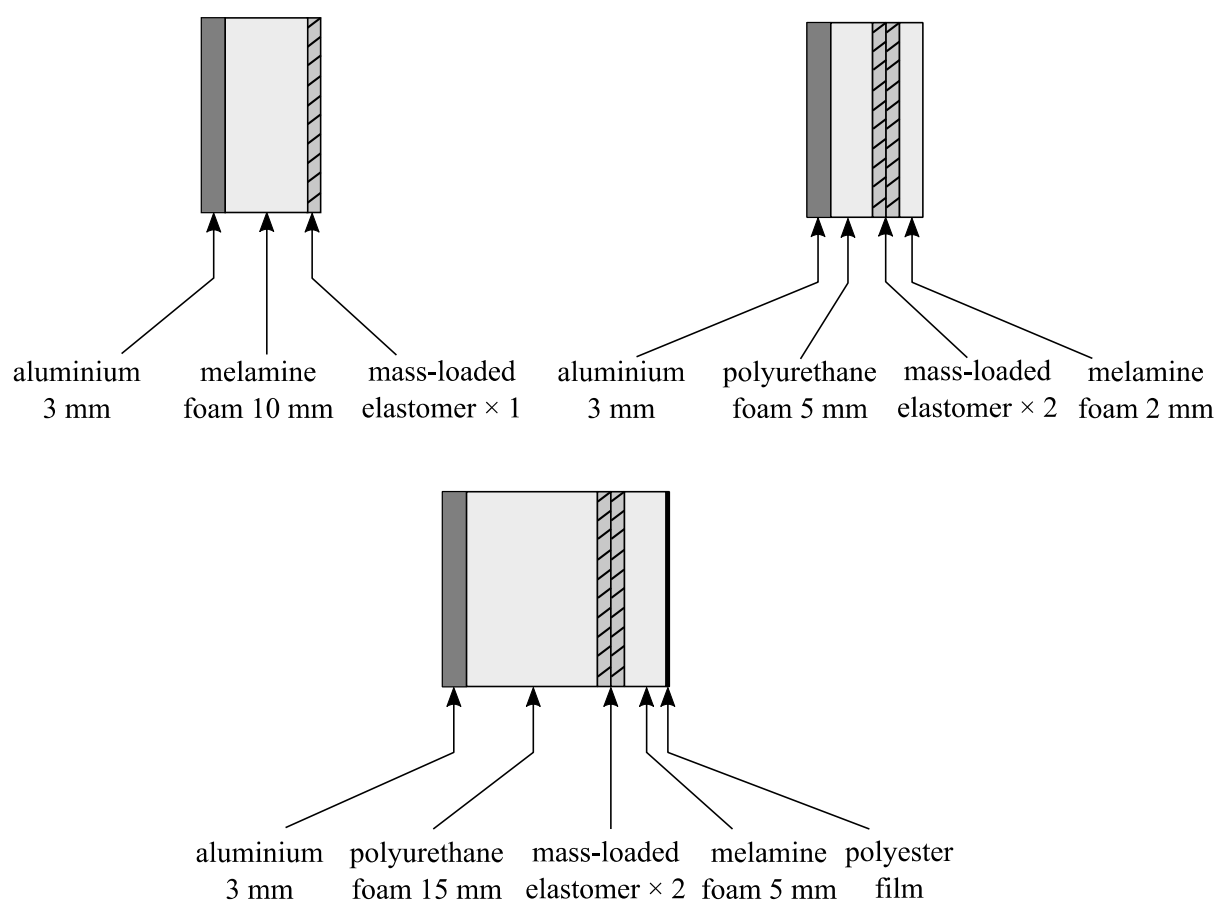

Figure 2: Examples of multilayer structures used on aluminium base plate for the case study

Following these design rules, identified topology categories and the material list, a total of 4951 different structures can be assembled. From the given set of materials, requirements must be met for any chosen situation, involving different performance goals. As discussed above, the usual tools used by multilayer package designers and researchers do not have the ability to solve the case. Therefore, a new tool is presented below for multilayer acoustic package selection.

\section{New tool for assessing performance}

Most of the usual methods for selecting the "best" multilayer acoustic package are far from practical since they concentrate on a few possible solutions. From the works of Ashby [22] and Sirisalee et al. [18], a tool for assessing performance is developed. The intent is to provide a global view of solutions, along with their benefits and drawbacks.

\subsection{Need for a database}

The new tool must consider all possible solutions, so as to allow a compromise between performance objectives. Moreover, it has to quickly adapt to various requirements, without needing additional computation time when changing the design preferences. Therefore, for all possible combinations, the following performance parameters are pre-computed and gathered in a database:

- transmission loss (TL) for third-octave frequency bands from $50 \mathrm{~Hz}$ to $5000 \mathrm{~Hz}$

- absorption coefficient ( $\alpha$ ) for third-octave frequency bands from $50 \mathrm{~Hz}$ to $5000 \mathrm{~Hz}$ 
- Sound Transmission Class (STC), a single-number rating used to evaluate the sound insulation of a structure, as defined in ASTM E413-16 [34]

- Sound Absorption Average (SAA), a single-number rating corresponding to the average of the sound absorption coefficient over the third octave frequency bands from 200 to $2000 \mathrm{~Hz}$, as defined in ASTM C423-17 [35]

- total thickness of the multilayer package $(h)$

- total surface density of the multilayer package $\left(\rho_{\mathrm{S}}\right)$

\subsection{Selecting the best solutions}

The many solutions from the database can be displayed in various ways. By selecting three performance indicators, it is possible to display the data on a three-dimensional scatter plot. This allows the designer to have a global view of the ranges of values for performance indicators. Projections on planes can be made to improve readability.

Sirisalee et al. [18] proposed a multi-criteria material selection method, which consists in two steps: first determining the Pareto set, then displaying the "better" solutions from the Pareto set in a trade-off chart.

For given performance parameters, the Pareto set puts forward the solutions with the best performances. Solutions are sorted into two groups: dominated solutions and non-dominated solutions. Solution A is dominated by solution B if the two following conditions are verified:

- For all performance parameters, A is matched or outperformed by B;

- There is at least one parameter for which A is strictly outperformed by B.

Non-dominated solutions form the Pareto set, as illustrated in Figure 3 for two performance parameters $P_{1}$ and $P_{2}$. Thus, the Pareto set is a way to filter solutions, only keeping the more interesting solutions: if a solution is part of the Pareto set, no other solution exists which improves a performance parameter without deteriorating another performance parameter. In the following section, a trade-off tool is presented, which allows selecting one solution among the Pareto set solutions. 
Dominated solutions

Non-dominated solutions

(Pareto set)

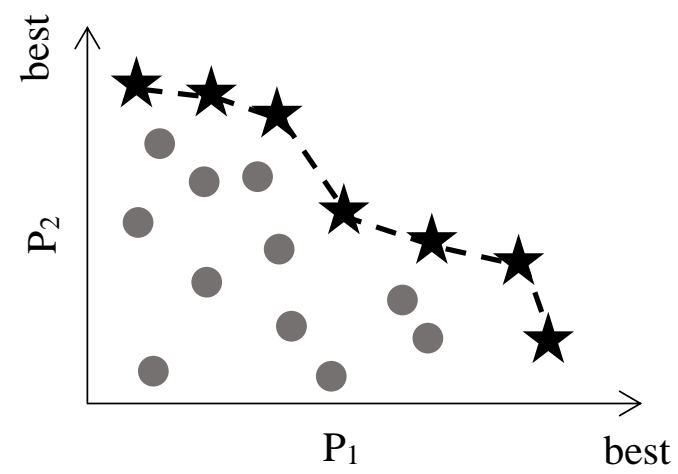

Figure 3: Illustration of the Pareto set for two performance parameters

\subsection{Utility function and acoustic trade-off chart}

All solutions in the Pareto set may be considered good solutions, depending on the relative importance of all performance parameters. Sirisalee et al. [18] introduced a utility function $Z$, allowing a designer to categorize solutions depending on the weight (or the importance) given to each performance parameter $P_{i}$, for a set of $n$ performance parameters:

$$
Z=\sum_{i=0}^{n-1} \pm E_{i} P_{i}
$$

$E_{i}$ are called "exchange constants" and are user defined, according to the preference given to each performance parameter. They depend on the product specifications and are greatly based on the designers' experience. The solution which minimizes $Z$ for a chosen set of exchange constants is the best solution. A plus sign is used in front of parameters that must be minimized, while a minus sign is used in front of parameters that must be maximized. This generalizes the expression from Sirisalee where all parameters were minimized.

One of the parameters $P_{0}$ can serve as a reference, setting $E_{0}=1$ : hence, $E_{i}$ expresses the weight of $P_{i}$ relative to $P_{0}$. The utility function becomes:

$$
Z= \pm P_{0}+\sum_{i=1}^{n-1} \pm E_{i} P_{i}
$$

In the particular case where there are 3 performance parameters, the problem can be illustrated by an exchange-constant chart. For each set of exchange constants $\left(E_{1}, E_{2}\right)$, the utility function is calculated for each solution of the Pareto set and the "best solution" is the one with the lowest $Z$. This "best solution" is 
thus displayed on the chart at position $\left(E_{1}, E_{2}\right)$. Therefore, this chart is a "map" showing the best solution for each set $\left(E_{1}, E_{2}\right)$. Thus, by weighting performance parameters with respect to a reference performance parameter, it is possible to easily select the appropriate solution to a given problem. The choice of a set $\left(E_{1}, E_{2}\right)$ allows selecting one single solution on the chart. This concept was used by Sirisalee et al. [18] for general material selection in engineering design. Application to the design of multilayer acoustic packages allows a designer to make a compromise between three chosen performance parameters (among those presented in section 4.1) through an "acoustic trade-off chart". Figure 4 shows an example of layout for the acoustic trade-off chart. For this example, surface density is taken as the reference $P_{0}$, STC as $P_{1}$ and SAA as $P_{2}$. The choice for $P_{0}, P_{1}$ and $P_{2}$ is made by the designer and depends on the specifics of the project. Solutions appear as clearly distinct areas, forming a "map". Each set of coordinates $\left(E_{1}, E_{2}\right)$ corresponds to a position on the chart and to a single multilayer acoustic package. In the given example, if the designer prefers good absorption and transmission over a low surface density, the suitable solutions are $\mathrm{b}$ and $\mathrm{c}$. In the case where low surface density is most important, solution $\mathrm{g}$ is most suitable.

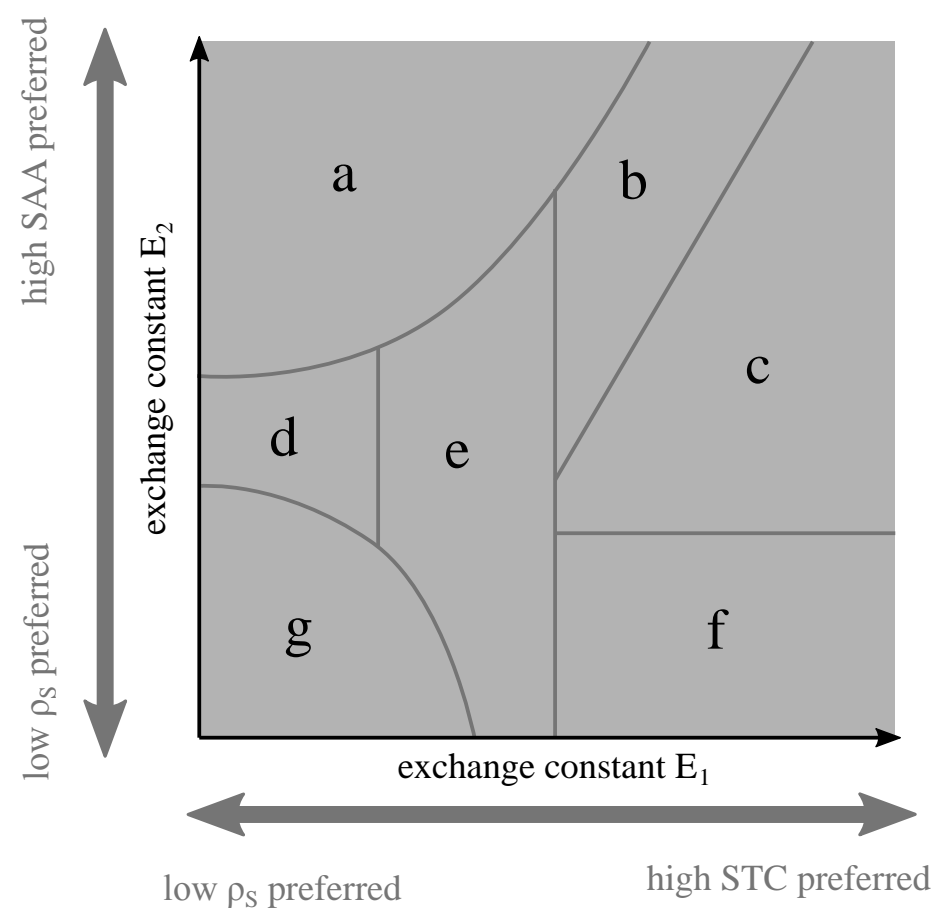

Figure 4: Example of acoustic trade-off chart for three parameters $\mathrm{P}_{0}=\rho_{\mathrm{S}}, \mathrm{P}_{1}=\mathrm{STC}$ and $\mathrm{P}_{2}=\mathrm{SAA}$

Often in practice, more than three performance parameters need to be considered, which cannot be displayed on a single trade-off chart. In this case, using several acoustic trade-off charts would allow designers to assess the appropriate solution. Software tools may be needed to simultaneously display the various charts as the designer is adjusting the exchange constants. 


\section{Application of the new tool to the case study}

\subsection{Overview of the solutions}

All combinations of layers from the case study presented in 3 are calculated, and the results (transmission loss, absorption coefficient, STC, SAA, total thickness and total surface density) are stored in a database. Once the database is complete, it can serve in many cases of acoustic package design without additional calculation.

A total of 4951 multilayer acoustic packages have been calculated. Figure 5 shows the database solutions displayed in a scatter plot with respect to their SAA, STC and surface density. For improved legibility, flat projections of the scatter plots are also presented on Figure 6. This scatter plot is analogous to material properties charts for material selection in mechanical design [22]. Each computed structure is represented by a point, whose coordinates are the corresponding performance. The solutions cover a large range of surface densities $\left(8.1\right.$ to $40.5 \mathrm{~kg} / \mathrm{m}^{2}$ ), SAA (0.01 to 0.77) and STC (20 to 37). A color code identifies solutions by structure categories (defined in Table 2 and Figure 1). Although performance varies a lot within each category, some trends can be discerned. For example, solutions from category A present low surface density (under $10 \mathrm{~kg} / \mathrm{m}^{2}$ ) and can reach high SAA (up to 0.75). Solutions from category F present low SAA (under 0.10) and cover large ranges of STC and surface densities. These trends can help designers understand the effectiveness of different categories of acoustic packages. However, it is still hard to make a choice among all the displayed solutions. Suitable solutions for the case study are scattered among other less interesting solutions.

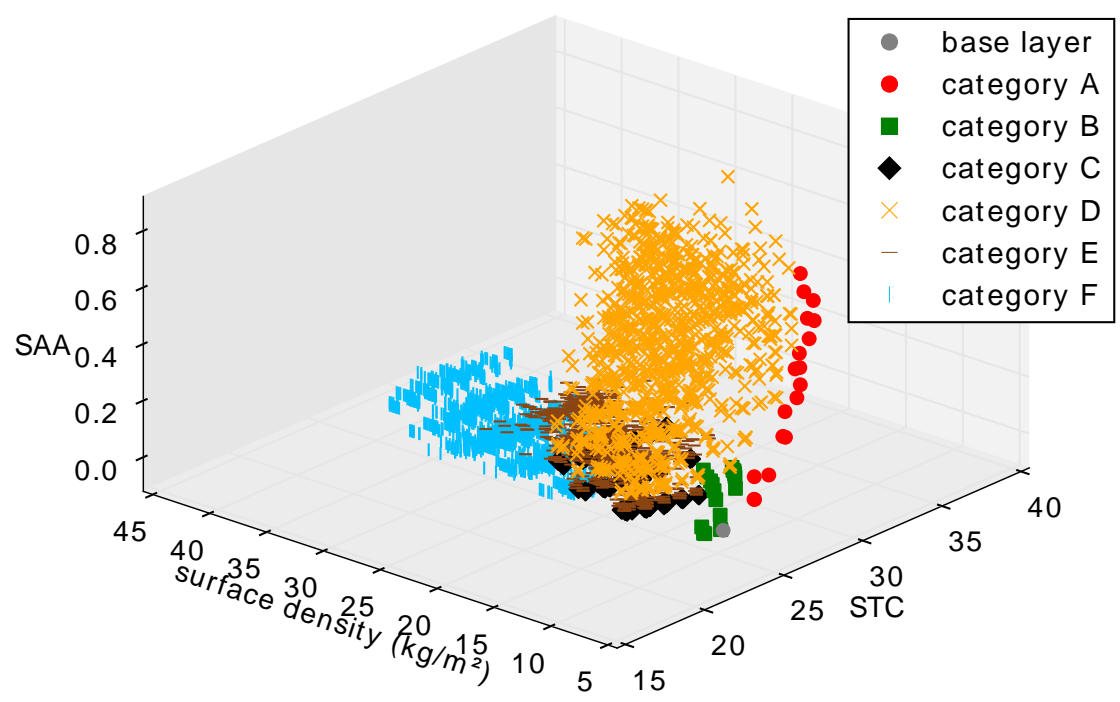

Figure 5: Three-dimensional scatter plot displaying solutions according to their SAA, STC and surface density 
(a)

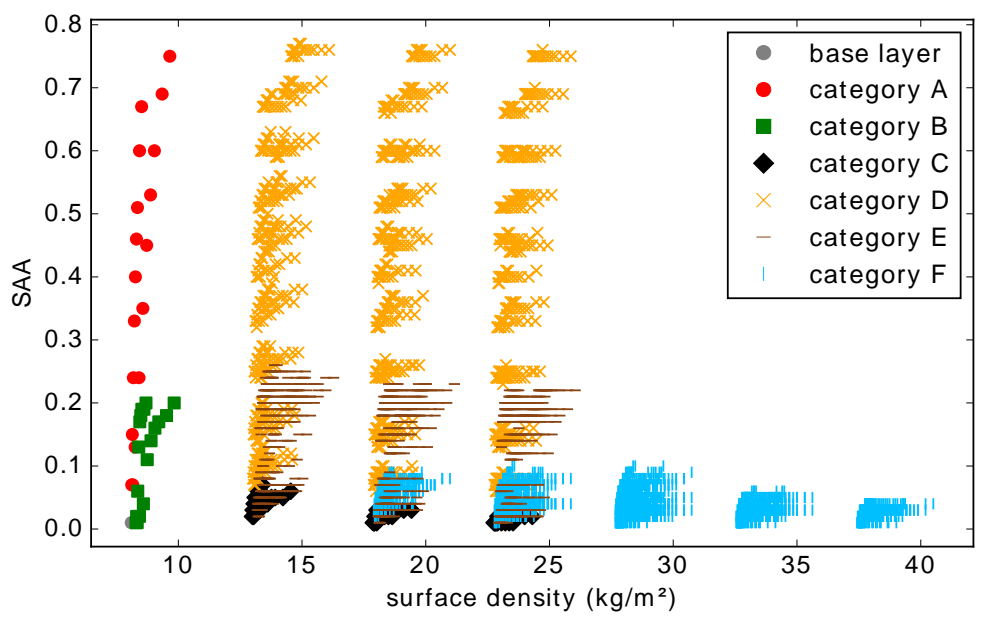

(b)

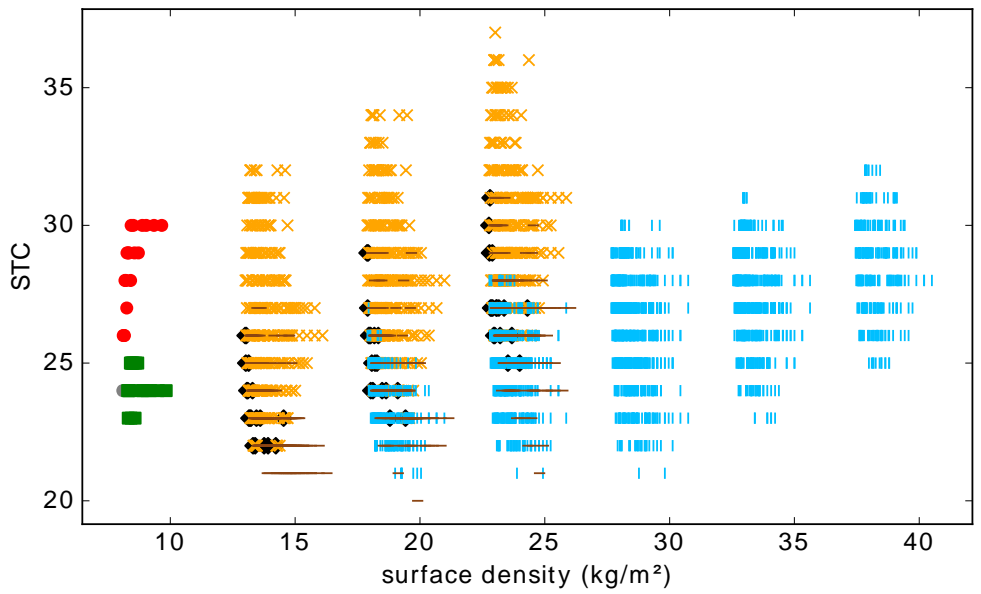

(c)

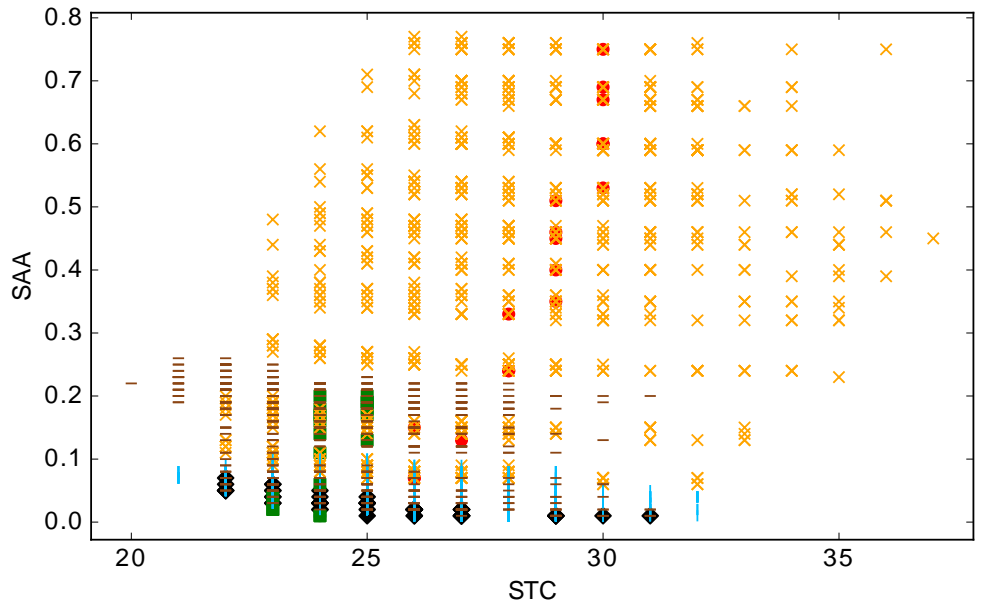

Figure 6: Flat projections of the scatter plot: (a) SAA versus surface density, (b) STC versus surface density, (c) SAA versus STC 
By selecting only the "better solutions", the Pareto set gets rid of all "less interesting" solutions. From the scatter plot, the Pareto set is calculated and displayed, as shown on Figure 7. Solutions belonging to the Pareto set are represented by stars. From the 4951 solutions, only 41 non-dominated solutions are kept. The Pareto set can be interpreted as the side of the plot where performance is best (low surface density, high SAA, high STC). Once the Pareto set is defined, the last step is to choose one solution among all "better solutions" using the trade-off chart introduced in section 4.3.

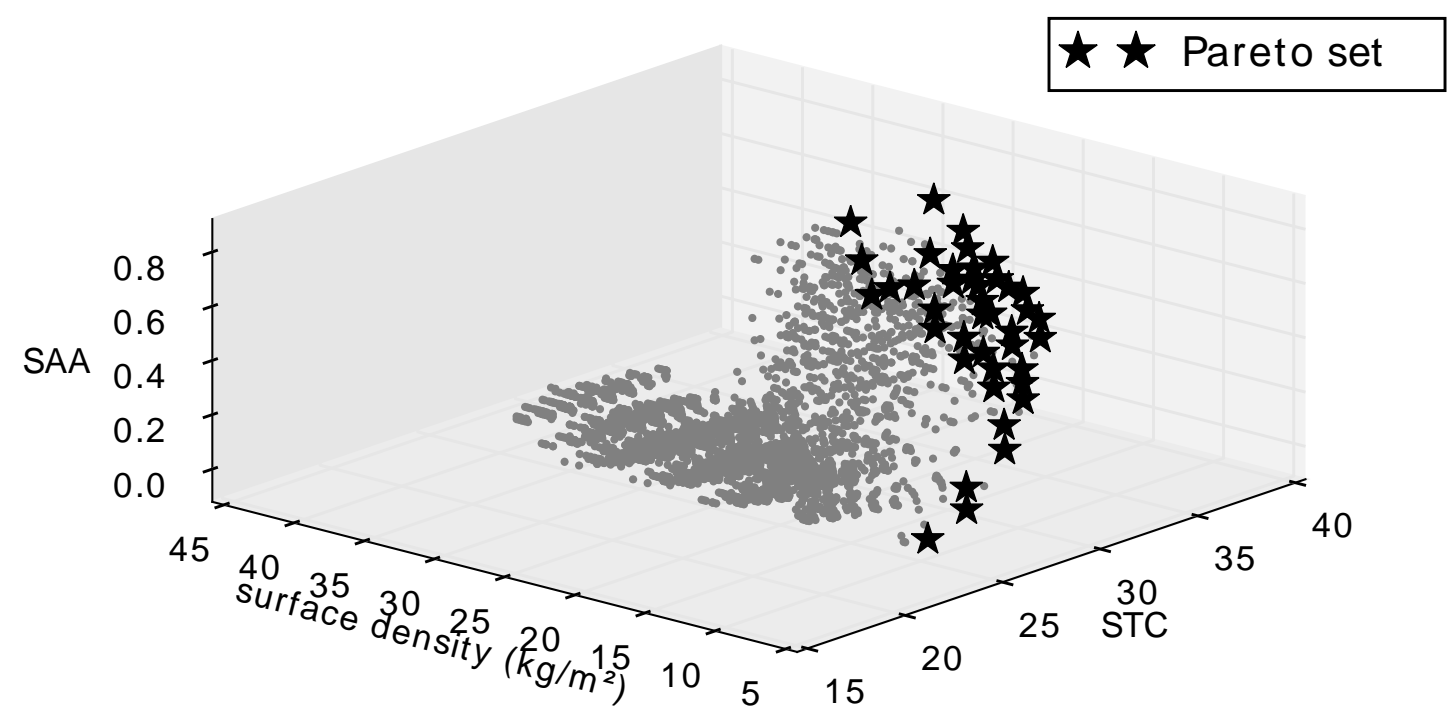

Figure 7: Display of the Pareto set

\subsection{Trade-off chart}

The acoustic trade-off chart aims at helping a designer to choose one solution among the Pareto set, while dealing with conflicting objectives. As examples to illustrate the use of the chart, two situations are presented in the following:

- Situation 1: sound insulation of an automotive panel with an emphasis put on a broad-band transmission loss and absorption coefficient

- $\quad$ Situation 2: sound package for mitigating a sound peak at $1000 \mathrm{~Hz}$

\subsubsection{Situation 1}

This situation corresponds to the case where broad-band insulation is needed, in the context of an automotive panel (e.g. car firewall). Three performance parameters are chosen: surface density, STC and SAA. The last two are particularly adapted to broad-band noise problem, since they are defined over large frequency ranges. The utility function is defined as the following:

$$
Z=\rho_{S}-E_{1} S T C-E_{2} S A A
$$


Surface density is defined as the reference parameter. STC and SAA values are preceded by a minus sign: thus, increasing STC and SAA or decreasing surface density (which is profitable) results in a lower Z, therefore bringing a "better solution".

The utility function is calculated for $E_{1}$ ranging from $10^{-4}$ to $10^{4}$ and for $E_{2}$ ranging from $10^{-4}$ to $10^{4}$, and each axis is divided into 300 steps. Without prior experience with the trade-off chart, a few tries may be necessary to identify the right limit values and number of steps. The following criteria are used as a guide for selecting the limit values and the number of steps:

- The limit values for $E_{1}$ and $E_{2}$ are chosen to make all solutions visible.

- The number of steps is chosen to allow a good graphic resolution, so that no applicable solution is hidden.

As all properties are pre-computed in the database, the computational cost associated with iteratively adjusting the limits and the number of steps is low. Depending on the number of structures in the database and the number of steps, it ranges from a few seconds to a few minutes.

The resulting acoustic trade-off chart is displayed on Figure 8. The trade-off chart is plotted using an inhouse program developed in Python. It puts forward 16 solutions from the Pareto set along with their performance, where categories are distinguished by colors. Each area delimited by a line corresponds to a given stacking. The chart brings out the fact that some categories are "better" than other at achieving certain performance. The base layer (no acoustic treatment) stands in the bottom-left corner, because it is the solution which minimizes the surface density. Category $\mathrm{D}$ (base layer + porous layer + barrier + porous layer) is more efficient when high STC or high SAA is the most wanted parameter. Category A (base layer + porous layer) is more efficient when a reasonable balance between all three parameters is needed. Other categories do not appear on this chart because for this situation, they are not part of the Pareto set. In particular, category $\mathrm{F}$ presents a double mass-air-mass resonance which lowers the transmission loss at given frequencies, and consequently its low STC excludes it from the Pareto set.

This chart also allows identifying one solution for a given compromise. Table 3 shows two solutions obtained for $\left(E_{1}, E_{2}\right)=(100,1)$ and $\left(E_{1}, E_{2}\right)=(1,100) . E_{1}=100$ and $E_{2}=1$ mean high STC is given high priority over low surface density, and high SAA is given the same priority as low surface density, which leads to a package in category D. On the other hand, $E_{1}=1$ and $E_{2}=100$ mean high STC is given the same priority as low surface density, and high SAA is given high priority over low surface density, which leads to a package in category A. 


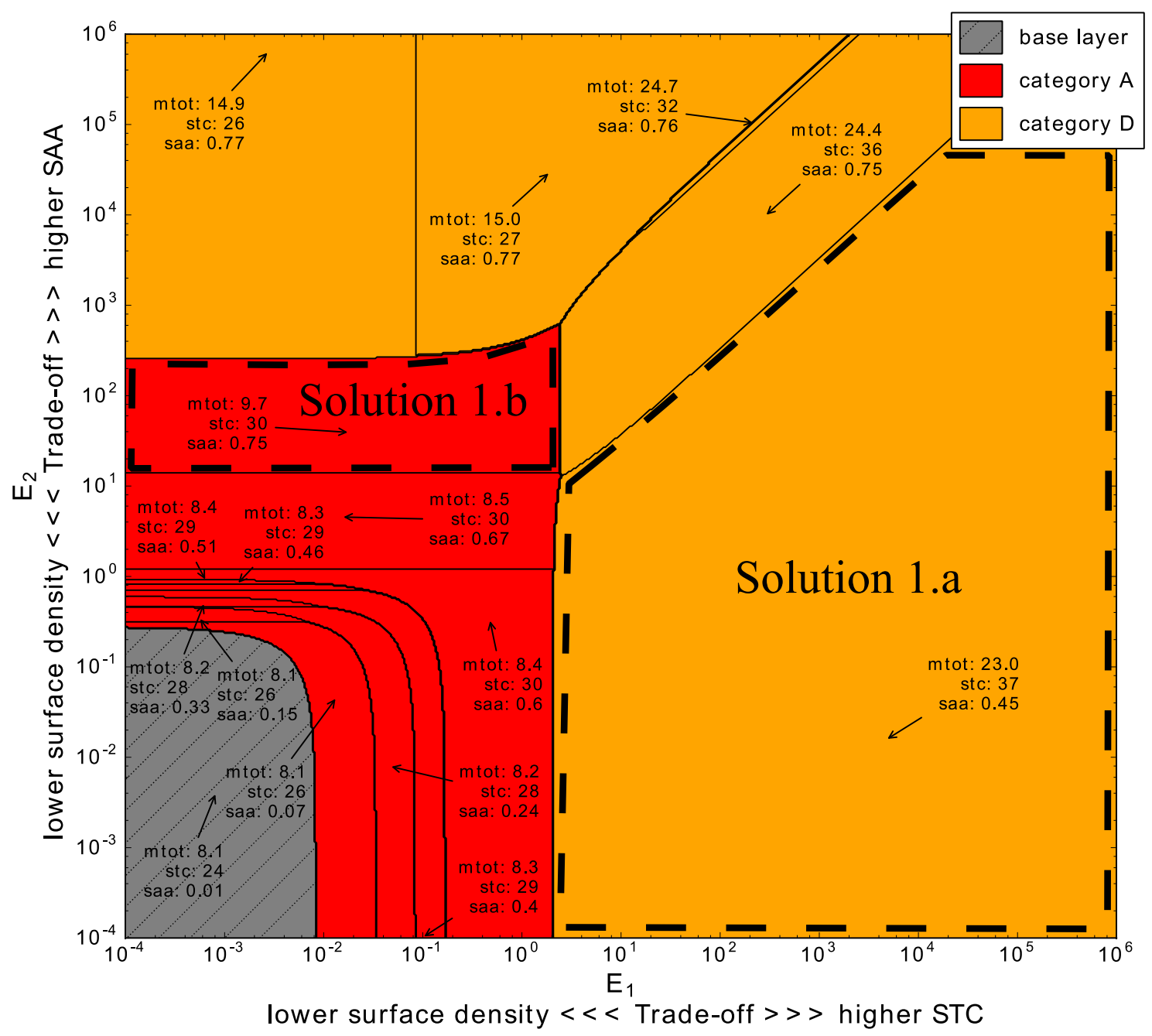

Figure 8: Acoustic trade-off chart displaying the Pareto solutions for Situation 1. The choice of a solution can be made by a trade-off between mass density of the package, STC and SAA 
Table 3: Solutions for Situation 1

\begin{tabular}{|c|c|c|c|c|c|}
\hline \multirow[b]{2}{*}{ Solution 1.a } & \multirow{2}{*}{$\begin{array}{c}E_{1} \\
100\end{array}$} & \multirow{2}{*}{$\begin{array}{c}E_{2} \\
1\end{array}$} & \multirow{4}{*}{\begin{tabular}{ll} 
& \multicolumn{1}{c}{ topology } \\
- & aluminium $3 \mathrm{~mm}$ \\
- & polyurethane foam $2 \mathrm{~mm}$ \\
- & mass-loaded elastomer $\times 3$ \\
- & melamine foam $25 \mathrm{~mm}$
\end{tabular}} & \multicolumn{2}{|c|}{ performance } \\
\hline & & & & $\rho_{\mathrm{S}}\left(\mathrm{kg} \cdot \mathrm{m}^{-2}\right)$ & 23.02 \\
\hline & & & & STC & 37 \\
\hline & & & & SAA & 0.45 \\
\hline \multirow[t]{3}{*}{ Solution 1.b } & 1 & 100 & - $\quad$ aluminium $3 \mathrm{~mm}$ & $\rho_{\mathrm{S}}\left(\mathrm{kg} \cdot \mathrm{m}^{-2}\right)$ & 9.66 \\
\hline & & & - polyurethane foam $50 \mathrm{~mm}$ & STC & 30 \\
\hline & & & & SAA & 0.75 \\
\hline
\end{tabular}

\subsubsection{Situation 2}

This situation corresponds to the case where a sound package is needed to mitigate a sound peak at $1000 \mathrm{~Hz}$. This can happen when a part or a mechanism resonates at this particular frequency. Controlling transmission loss and absorption coefficient in the $1000 \mathrm{~Hz}$ frequency band is more adapted to this problem than controlling STC and SAA. Thus, the utility function is defined as the following:

$$
Z=\rho_{S}-E_{1} T L_{1000}-E_{2} \alpha_{1000}
$$

where $T L_{1000}$ is the transmission loss at $1000 \mathrm{~Hz}$ and $\alpha_{1000}$ is the absorption coefficient at $1000 \mathrm{~Hz}$.

The utility function was calculated for $E_{1}$ ranging from $10^{-4}$ to $10^{4}$ and for $E_{2}$ ranging from $10^{-4}$ to $10^{4}$, and each axis divided into 300 steps. The resulting acoustic trade-off chart is displayed on Figure 9.

Table 4 shows two solutions obtained for $\left(E_{1}, E_{2}\right)=(100,1)$ and $\left(E_{1}, E_{2}\right)=(0.1,200) . E_{1}=100$ and $E_{2}=1$ mean high TL is given high priority over low surface density, and high $\alpha$ is given the same priority as low surface density, which leads to a package in category $\mathrm{F}$ (base layer + porous layer + barrier + porous layer + facing). This solution, which includes two stacks of three mass-loaded elastomer barriers, is particularly heavy and presents high transmission loss at $1000 \mathrm{~Hz}(78.8 \mathrm{~dB})$.

$E_{1}=0.1$ and $E_{2}=200$ mean high TL is given lower priority than low surface density, and high $\alpha$ is given high priority over low surface density, which leads to a package in category $\mathrm{D}$ (base layer + porous layer + barrier + porous layer). In solution 2.b, the high coefficient absorption (0.91) at $1000 \mathrm{~Hz}$ is explained by the thick layers of porous materials in the package. 


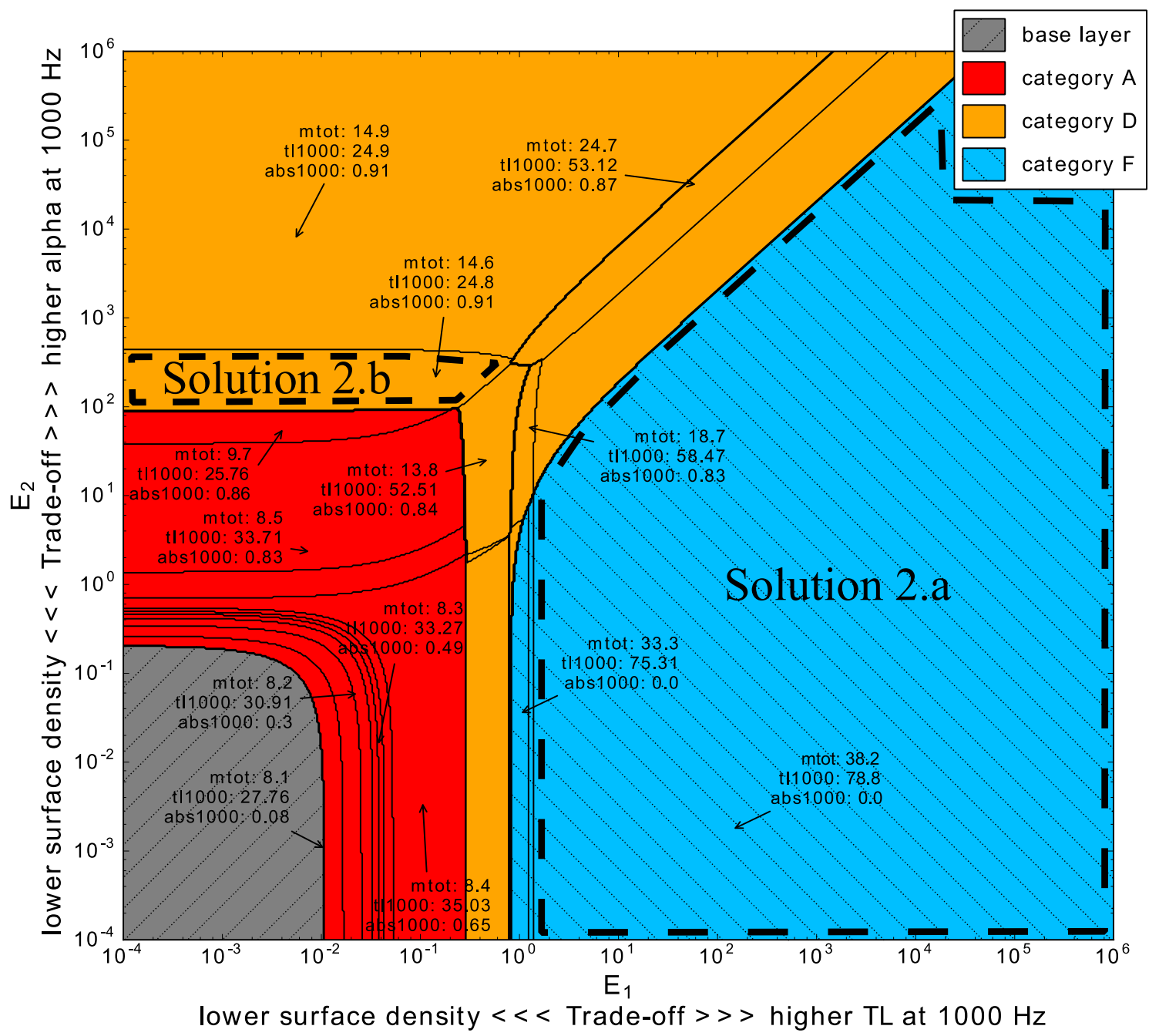

Figure 9: Acoustic trade-off chart displaying the Pareto solutions for Situation 2. The choice of a solution can be made by a trade-off between mass density of the package, TL and $\alpha$ 
Table 4: Solutions for Situation 2

\begin{tabular}{|c|c|c|c|c|c|}
\hline \multirow[b]{2}{*}{ Solution 2.a } & \multirow{2}{*}{$\begin{array}{c}E_{1} \\
100\end{array}$} & \multirow{2}{*}{$\frac{E_{2}}{1}$} & \multirow{2}{*}{\begin{tabular}{ll} 
& \multicolumn{1}{c}{ topology } \\
- & aluminium $3 \mathrm{~mm}$ \\
- & melamine foam $50 \mathrm{~mm}$ \\
- & mass-loaded elastomer $\times 3$ \\
- & melamine foam $50 \mathrm{~mm}$ \\
- & Mass-loaded elastomer $\times 3$
\end{tabular}} & \multicolumn{2}{|c|}{ performance } \\
\hline & & & & $\begin{array}{c}\rho_{\mathrm{S}}\left(\mathrm{kg} \cdot \mathrm{m}^{-2}\right) \\
T L_{1000}(\mathrm{~dB}) \\
\alpha_{1000}\end{array}$ & $\begin{array}{c}38.23 \\
78.8 \\
0\end{array}$ \\
\hline Solution 2.b & 0.1 & 200 & $\begin{array}{ll}\text { - } & \text { aluminium } 3 \mathrm{~mm} \\
\text { - } & \text { melamine foam } 50 \mathrm{~mm} \\
\text { - } & \text { mass-loaded elastomer } \times 1 \\
\text { - } & \text { polyurethane foam } 50 \mathrm{~mm}\end{array}$ & $\begin{array}{c}\rho_{\mathrm{S}}\left(\mathrm{kg} \cdot \mathrm{m}^{-2}\right) \\
T L_{1000}(\mathrm{~dB}) \\
\alpha_{1000}\end{array}$ & $\begin{array}{r}14.58 \\
24.83 \\
0.91\end{array}$ \\
\hline
\end{tabular}

\subsection{Case study discussion}

In both situations, the acoustic trade-off chart provides a clear overview of possible solutions from the Pareto set, along with trade-off guidelines. Noteworthy in both situations, only three or four categories of multilayer packages are represented, as the other categories are not selected by the Pareto set and the utility function. For some acoustic problems, a few categories only may be suitable, with variations in materials and thicknesses. This single piece of information can lead the designer toward the most efficient categories of multilayer acoustic packages. The chart also puts forward the fact that an increase in mass does not always lead to higher STC. Indeed, solutions from category F, although they are heavier, do not arise when STC is a performance parameter. This is because using two loaded barriers leads to two mechanical resonances, which decreases transmission loss at specific frequencies, and results in a lower STC.

The choice of exchange constants $E_{1}$ and $E_{2}$ depends on the priority given by a designer to each performance parameter. Through this choice, a solution can be assigned to the problem. For illustration purposes only, specific values of the exchange parameters were presented in this case study, leading to the selection of one solution for each case. Still, the sensitivity of the solutions should be given careful consideration: in some cases, a small change in the choice of exchange parameters may lead to entirely different solutions, and this should be taken into account in the design process. The charts give visual information on the sensitivity of solutions to parameter choice through the area occupied by each solution. Solutions 1.a and 2.a cover large areas on their respective charts: therefore, the obtained solutions will not change for different but similar exchange parameters, and they can be selected with confidence. If, instead of $(100,1)$, the exchange parameters had been $(50,0.5)$ or even $(1000,1)$, the same solutions would have been identified. On the other hand, solutions 1.b and 2.b occupy smaller areas on their respective charts, and changing the value of $E_{2}$ in particular may lead to different solutions. In this case, the designer may consider a range of exchange parameters, corresponding to some adjacent solutions which may also be worth investigating. One major advantage of this chart is its ability to represent close alternative solutions next to the assigned solution. This capability also allows taking into account other constraints, such as cost or availability of the materials. 


\section{Conclusion}

A new design tool has been presented, which can be used as a design guide at the early stage of multilayer acoustic packages design. From a set of available layers and design constraints, a database of possible multilayer structures is calculated. Best solutions from the Pareto set are selected using a utility function and displayed on a designer-oriented acoustic trade-off chart. This chart allows for a quick visual analysis of suitable solutions. It represents the necessary trade-offs between conflicting performance goals: each displayed solution is a "best solution", as defined by the Pareto set, and a designer must prioritize the performance parameters in order to reach their design goal.

The design tool was applied to a case study on acoustic packages representative of automotive applications, composed of foam layers, barriers and facings stacked and bonded to an aluminium base panel. Acoustic trade-off charts were plotted for two situations: one in which surface density, STC and SAA were chosen as performance parameters, and another in which performance parameters were the surface density of the multilayer package, absorption coefficient and transmission loss for a given frequency range. In both situations, from an initial database of 4951 possible solutions, trade-off charts successfully identified suitable solutions to example cases, where relative importance between parameters were defined through exchange constants.

While the case study used the Transfer Matrix Method to predict the properties of the structures, the proposed design tool uses the prediction method as a black box which relates entry parameters to performance parameters. Therefore, other material models or performance parameters could be investigated as needed without modifying the design tool itself. This also means that the obtained solutions are valid providing the prediction method is valid: designers have to select an appropriate prediction method for the problem studied based on their experience or based on previous numerical or experimental validations.

The strength of this methodology is its flexibility in terms of objectives and the fact that it proposes many suiting solutions with variations, instead of a single optimized solution. The display of solutions is intended to be effective and visual for the designer. Therefore, it is suited for multi-objective design of multilayer acoustic packages in an industrial context. The format involving three performance parameters is ideal for a clear visual display. With technological assistance, more than three parameters could be used as preference parameters, and trade-off would include all aspects of the acoustic package. The tool can also be easily modified to include additional performance parameters such as cost, for instance, which is a decisive design parameter. The use of this design tool could lead to significant gains of time at the early stage of design of acoustic packages, when it is most crucial.

\section{Acknowledgements}

The authors wish to recognize the National Sciences and Engineering Research Council of Canada (NSERC) and Elasto Proxy Inc. for their financial support. They also thank Elasto Proxy Inc. for providing technical expertise and materials for this research work. 


\section{References}

[1] A.-M. Fillet, "Ninth international congress on noise as a public health problem," Environnement, Risques \& Santé, vol. 8, no. 1, pp. 70-74, 2009.

[2] Y.-T. Tsai, S. J. Pawar, and J. H. Huang, "Optimizing material properties of composite plates for sound transmission problem," Journal of Sound and Vibration, vol. 335, pp. 174-186, 2015.

[3] R. Cherif, A. Wareing, and N. Atalla, "Evaluation of a hybrid TMM-SEA method for prediction of sound transmission loss through mechanically coupled aircraft double-walls," Applied Acoustics, vol. 117, pp. 132-140, 2017.

[4] C.-C. Liao, C.-W. Hsueh, T.-Y. Zhong, C.-C. Hsu, and Y.-K. Fuh, "Multiple vibrating plates of sound suppression mask for minimizing tics impulse acoustics of Tourette's patients," Applied Acoustics, vol. 129, pp. 27-35, 2018.

[5] J. F. Allard and N. Atalla, "Modelling multilayered systems with porous materials using the transfer matrix method," in Propagation of Sound in Porous Media: Modelling Sound Absorbing Materials, Second Edition, 2009, pp. 243-281.

[6] O. Doutres and N. Atalla, "Experimental estimation of the transmission loss contributions of a sound package placed in a double wall structure," Applied Acoustics, vol. 72, no. 6, pp. 372-379, 2011.

[7] A. Dijckmans, G. Vermeir, and J. W. Niggebrugge, "Optimization of the acoustic performances of lightweight sandwich roof elements," in INTER-NOISE, 2009.

[8] R. H. Lyon, Statistical energy analysis of dynamical systems: theory and applications. 1975.

[9] F. J. Fahy and P. Gardonio, Sound and structural vibration: radiation, transmission and response. Academic press, 2007.

[10] F. C. Sgard, X. Olny, N. Atalla, and F. Castel, "On the use of perforations to improve the sound absorption of porous materials," Applied Acoustics, vol. 66, no. 6, pp. 625-651, 2005.

[11] W.-H. Chen, F.-C. Lee, and D.-M. Chiang, "On the acoustic absorption of porous materials with different surface shapes and perforated plates," Journal of Sound and Vibration, vol. 237, no. 2, pp. 337-355, 2000.

[12] J. J. del Coz Díaz, F. P. Álvarez Rabanal, P. J. García Nieto, and M. A. Serrano López, "Sound transmission loss analysis through a multilayer lightweight concrete hollow brick wall by FEM and experimental validation," Building and Environment, vol. 45, no. 11, pp. 2373-2386, 2010.

[13] M. H. Fouladi, M. J. M. Nor, M. Ayub, and Z. A. Leman, "Utilization of coir fiber in multilayer acoustic absorption panel," Applied Acoustics, vol. 71, no. 3, pp. 241-249, 2010.

[14] C. J. Naify, C.-M. Chang, G. McKnight, and S. Nutt, "Transmission loss and dynamic response of membrane-type locally resonant acoustic metamaterials," Journal of Applied Physics, vol. 108, no. $11,2010$.

[15] T. Yamamoto, S. Maruyama, S. Nishiwaki, and M. Yoshimura, "Thickness optimization of a multilayered structure on the coupling surface between a structure and an acoustic cavity," Journal of Sound and Vibration, vol. 318, no. 1, pp. 109-130, 2008. 
[16] O. Tanneau, J. B. Casimir, and P. Lamary, "Optimization of multilayered panels with poroelastic components for an acoustical transmission objective," The Journal of the Acoustical Society of America, vol. 120, no. 3, pp. 1227-1238, 2006.

[17] J. Zhou, A. Bhaskar, and X. Zhang, "Optimization for sound transmission through a double-wall panel," Applied Acoustics, vol. 74, no. 12, pp. 1422-1428, 2013.

[18] P. Sirisalee, M. F. Ashby, G. T. Parks, and P. J. Clarkson, "Multi- Criteria Material Selection in Engineering Design," Advanced Engineering Materials, vol. 6, no. 1- 2, pp. 84-92, 2004.

[19] C. J. Cameron, E. Lind Nordgren, P. Wennhage, and P. Göransson, "On the balancing of structural and acoustic performance of a sandwich panel based on topology, property, and size optimization," Journal of Sound and Vibration, vol. 333, no. 13, pp. 2677-2698, 2014.

[20] M. Inoue, Y.-E. Nahm, and H. Ishikawa, "Application of preference set-based design method to multilayer porous materials for sound absorbency and insulation," International Journal of Computer Integrated Manufacturing, vol. 26, no. 12, pp. 1151-1160, 2013.

[21] P. Leite, M. Thomas, F. Simon, and Y. Bréchet, "Optimal Design of a Multifunctional Sandwich Panel With Foam Core: Lightweight Design for Flexural Stiffness and Acoustical Transmission Loss," Advanced Engineering Materials, vol. 17, no. 3, pp. 311-318, 2015.

[22] M. F. Ashby, "Chapter 4 - Material property charts," in Materials Selection in Mechanical Design (3rd Edition): Elsevier, 2005.

[23] M. Avalle, G. Chiandussi, and G. Belingardi, "Design optimization by response surface methodology: application to crashworthiness design of vehicle structures," Structural and Multidisciplinary Optimization, vol. 24, no. 4, pp. 325-332, 2002.

[24] M. M. Farag, Materials and process selection for engineering design. CRC Press, 2013.

[25] F. Fahy, "Chapter 11 - Transmission of Sound through Partitions," in Foundations of Engineering AcousticsLondon: Academic Press, 2001, pp. 315-351.

[26] J. P. Arenas and M. J. Crocker, "Recent trends in porous sound-absorbing materials," Sound \& vibration, vol. 44, no. 7, pp. 12-18, 2010.

[27] M. A. Biot, "Theory of propagation of elastic waves in a fluid- saturated porous solid. II. Higher frequency range," The Journal of the acoustical Society of america, vol. 28, no. 2, pp. 179-191, 1956.

[28] M. L. Munjal and P. T. Thawani, "Effect of protective layer on the performance of absorptive ducts," Noise Control Engineering Journal, vol. 45, no. 1, pp. 14-18, 1997.

[29] I. Yoshio and A. Tsuneo, "Studies of acoustical absorption of flexible polyurethane foam," Journal of Applied Polymer Science, vol. 27, no. 1, pp. 183-195, 1982.

[30] A. M. McNelis and W. O. Hughes, "Effect of Coversheet Materials on the Acoustic Performance of Melamine Foam," 2015.

[31] DuPont Teijin Films. (2003). Mylar ${ }^{\circledR}$ polyester film - Product Information. Available: http://usa.dupontteijinfilms.com/wp-content/uploads/2017/01/Mylar_Physical_Properties.pdf

[32] J. P. Parkinson, J. R. Pearse, and M. D. Latimer, "Sound absorption of elastic framed porous materials in combination with impervious films: effect of bonding," Applied Acoustics, vol. 63, no. 8, pp. 819-828, 2002. 
[33] K-FLEX USA. (2011). K-FONIK® $G K / G V$. Available: http://kflexusa.com/downloads/Technical\%20Data\%20Sheets/K-Flex\%20K-FONIK\%20GKGV.pdf

[34] E413-16 Classification for Rating Sound Insulation, 2016.

[35] C423-17 Standard Test Method for Sound Absorption and Sound Absorption Coefficients by the Reverberation Room Method, 2017. 